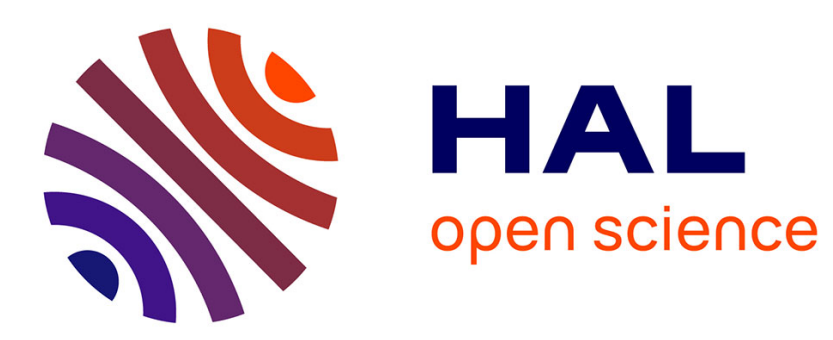

\title{
Les cultes impériaux dans les cités d'Asie Mineure: des spécificités provinciales?
}

Gabrielle Frija

\section{To cite this version:}

Gabrielle Frija. Les cultes impériaux dans les cités d'Asie Mineure: des spécificités provinciales?. Kaiserkult in den Provinzen des Römischen Reichs, Sep 2014, Zurich, Suisse. 10.1515/9783110420876011. hal-01396569

\author{
HAL Id: hal-01396569 \\ https://hal.science/hal-01396569
}

Submitted on 14 Nov 2016

HAL is a multi-disciplinary open access archive for the deposit and dissemination of scientific research documents, whether they are published or not. The documents may come from teaching and research institutions in France or abroad, or from public or private research centers.
L'archive ouverte pluridisciplinaire HAL, est destinée au dépôt et à la diffusion de documents scientifiques de niveau recherche, publiés ou non, émanant des établissements d'enseignement et de recherche français ou étrangers, des laboratoires publics ou privés. 


\title{
Les cultes impériaux dans les cités d'Asie Mineure: des spécificités provinciales?
}

\begin{abstract}
Cette étude porte sur l'existence de spécificités provinciales, en Asie Mineure, dans l'organisation des cultes impériaux civiques. Les cités et koina des provinces micrasiatiques organisent précocement des cultes d'Auguste et de ses successeurs. Si la compétition entre cités dans chaque province pour l'obtention des temples des koina a été bien étudiée, la comparaison entre les structures du culte à l'intérieur des différentes provinces restait à faire, particulièrement pour les cultes civiques. Sans revenir sur le problème des titres des dignitaires du culte provincial - asiarques, bithyniarques, lykiarques etc. - nous abordons ici la question du point de vue du rapport entre les cultes provinciaux et ceux des cités. En Asie, les cultes des cités sont pris en charge par des grands-prêtres locaux, les cités reproduisant à leur échelle l'organisation du culte provincial. En revanche, la diffusion de la grande-prêtrise civique semble moins systématique en Lycie, en Bithynie-Pont comme dans les provinces de l’Anatolie intérieure. L’Achaïe, enfin, étudiée ici à titre de comparaison, suit un modèle divergent puisque les cultes civiques précèdent les cultes fédéraux et provinciaux; la grande-prêtrise civique ne saurait donc y être considérée comme une imitation de la grande-prêtrise provinciale. Ainsi, du point de vue de l'organisation des cultes impériaux, les rapports entre l'assemblée fédérale et les cités diffèrent selon les provinces. En ce sens, on peut bien parler de spécificités provinciales: le cadre romain a finalement eu un effet sur l'adoption ou le rejet de certaines institutions, même lorsque cela n’entraîne pas la formation d'identités provinciales revendiquées.
\end{abstract}

The cities and koina of the microasiatic provinces of the Roman Empire organized cults for Augustus and his successors early on. The competition between cities to host provincial temples has been well studied, but the comparison of the structures of worship within the various provinces remained to be done, particularly with regard to civic cults. Leaving aside the variety of titles borne by the dignitaries of the provincial cult Asiarchs, Bithyniarchs, Lykiarchs -, we discuss the relationship between provincial and civic cults in each province. In Asia, civic cults were organized by local high priests, and reproduced provincial cults on a smaller scale. Civic high priesthood was less systematical in Lycia, in Bithynia et Pontus, as well as in other Anatolian provinces. In Achaia, by contrast, another model developed whereby civic cults preceded federal and provincial cults, and civic high priesthood emerged independently of provincial high priesthood. As far as the organization of imperial cults is concerned, the relationship between federal assemblies and cities therefore differed between provinces, making it possible for us to talk of provincial specificities. Structures and frontiers imposed by the Romans clearly had an effect on the adoption or rejection of certain institutions, even if this did not result in the formation of conscious provincial identities. 


\section{Introduction}

Les cultes impériaux, dans les provinces grecques, constituent un moyen de communication avec le pouvoir central, mais ont aussi un enjeu local très fort. L'exercice des charges liées au culte impérial a été un moyen, pour les élites locales et provinciales, de faire preuve à la fois de loyauté envers le prince et de générosité envers la communauté civique ou provinciale. Dans le monde compétitif des Grecs de l'époque impériale, le culte des empereurs est une occasion de plus d'exercer son évergétisme et d'affirmer sa domination sociale. ${ }^{1}$

Mais dans quelle mesure ce fonctionnement compétitif produit-il de la variété ou, au contraire, de l'homogénéité? Posée du point de vue du culte impérial, cette question touche au problème fondamental de l'unification religieuse dans l'empire romain. L'existence d'une religion impériale au sens propre est un non-sens dans un empire constitué de cités polythéistes autonomes ayant chacune leur panthéon. Pourtant, le culte des empereurs est bien un élément commun à tous les habitants de l'Empire. Dans quelle mesure est-il un facteur d'unification de l'espace impérial? Dans cet article, je me propose d'étudier cette vaste question non pas de façon synthétique, ni dans l'ensemble de l'empire, mais dans les provinces grecques en examinant si les cultes impériaux ont pris des physionomies différentes selon les provinces. Le cadre provincial me paraît particulièrement important au sens où il constitue un cadre administratif romain dans lequel se joue la compétition entre cités, c'est-àdire essentiellement entre élites civiques. Or, si les cultes impériaux connaissent des spécificités liées au cadre administratif romain, cela signifie que, dans une certaine mesure, ils constituent une activité religieuse détachée du monde civique. Si cette hypothèse s'avérait exacte, il faudrait les distinguer de la religion civique, non parce qu'ils seraient „grecs“ plutôt que „poliades“, mais parce qu'ils seraient le signe de l'existence de cultures provinciales.

Il ne s'agit pas seulement ici des cultes dits provinciaux, organisés par des assemblées de cités dans le cadre romain de la province, mais bien des cultes impériaux pratiqués dans les cités. Dans quelle mesure la forme que prend le culte impérial civique est-elle liée à l'appartenance provinciale? Les cultes civiques de Xanthos en Lycie, Milet en Asie et Héraclée dans le Pont diffèrent-ils en raison d'histoires locales, sont-ils tous semblables parce que tous grecs, ou bien diffèrent-ils parce que ces cités appartiennent à des provinces différentes? S’il existe des identités provinciales en matière de culte impérial, faut-il considérer ce phénomène religieux comme un phénomène supra-civique, bien qu'exercé dans le cadre traditionnel de la cité? Toutes ces questions sont liées au problème de la constitution d'identités provinciales intermé-

1 Sur le fonctionnement et la signification du culte impérial dans le monde grec, l'ouvrage de référence reste PRICE 1984. Pour des bilans historiographiques plus récents, voir GRADEL 2002, HARLAND 2003; pour l'Achaïe, KANTIRÉA 2007; CAMIA 2011; pour la province d'Asie, FRIJA 2012. 
diaires entre trois niveaux d'identité bien attestés: l'identité civique, cadre de la vie quotidienne; l'identité grecque; l'appartenance à un ensemble plus vaste, l'empire, au moins par la diffusion de la citoyenneté romaine. Il est possible qu'il faille ajouter un quatrième niveau d'identité, celui de la province, cadre dont les frontières sont pourtant souvent artificiellement imposées par les autorités romaines. L'enjeu est donc de réfléchir au problème de l'homogénéisation culturelle et religieuse de l'Empire en l'abordant du point de vue de possibles identités provinciales et en affinant la caractérisation générale de „grecs“ qui est certes indispensable, mais trop large pour décrire dans le détail la circulation et la diffusion des innovations de l'époque impériale. En effet, le culte impérial étant une nouveauté des débuts du principat augustéen, il a fallu inventer des formes pour organiser le culte; il s'agit ici d'étudier si ces formes ont circulé dans toutes les provinces grecques ou si elles sont restées confinées à un espace réduit.

Pour aborder ce vaste problème, je me suis concentrée sur un aspect précis des cultes impériaux, les sacerdoces des empereurs. En effet, grâce à plusieurs monographies sur le culte impérial dans les provinces du bassin égéen, nous disposons aujourd'hui de prosopographies et d'études systématiques permettant la comparaison. ${ }^{2}$ En outre, les prêtrises sont des éléments institutionnels concrets, tout à la fois produit et cadre de l'activité des élites provinciales: or ce sont ces élites qui, à mon sens, créent l'homogénéité ou la variété, en fonction des situations. Mais il faudrait mener l'étude pour les autres manifestations du culte impérial, concours, bâtiments et spectacles notamment. Je ne prétends donc pas résoudre la question posée en introduction, mais l'éclairer par l'étude comparée des sacerdoces du culte impérial dans les cités de plusieurs provinces anatoliennes et de l'Achaïe.

\section{L'Asie: l'influence politique et religieuse du koinon}

La province d'Asie est une création romaine dont les frontières ne correspondent à strictement parler à aucune entité politique antérieure. A l'époque augustéenne, elle dépasse largement le cadre de l'ancien royaume attalide. En outre, cette vaste province regroupe des territoires relativement hétérogènes, des grandes cités de la côte égéenne aux confins de la Phrygie et de la Galatie. ${ }^{3}$ Plusieurs déplacements de frontières provinciales ont légèrement modifié son étendue cours du Ier siècle. ${ }^{4}$ Mais un tableau à la fin du $\mathrm{II}^{\mathrm{e}}$ siècle $\mathrm{p}$. C. montre que, dans ce cadre relativement artificiel, les

2 Voir en particulier, pour l'Achaïe, KANTIRÉA 2007; REITZENSTEIN 2011; FRIJA 2012; FUJII 2013; le Pont-Bithynie n'a pas fait l'objet de monographie consacrée spécifiquement au culte impérial, mais le phénomène est étudié par MAREK 2003 et FERNOUX 2004.

3 Voir la synthèse récente de MAREK 2010; SARTrE 1997, 18; SARTRE 1998, 333-339.

4 Voir notamment le rattachement de la Pamphylie à la nouvelle province de Lycie en 43 p. C. 
cultes impériaux civiques fonctionnent de façon très homogène - en ce qui concerne les sacerdoces du moins. Partout ou presque, le culte impérial est assuré par des grands-prêtres (archiereis), parfois accompagnés d'une grande-prêtresse (archiereia), chargés du culte des Augustes dans leur ensemble. Les prêtres (hiereis) sont extrêmement rares aux $\mathrm{II}^{\mathrm{e}}$ et $\mathrm{III}^{\mathrm{e}}$ siècles: sur 219 prêtres et prêtresses attestés durant cette période, 153 sont des archiereis, dont 121 sans précision du culte dont ils sont chargés (les autres étant qualifiés de grands-prêtres „des Augustes“, „de la patrie“ ou encore „de la cité“); 46 des archiereiai, dont 39 sans mention de la divinité honorée. ${ }^{5}$ Sur les 219 personanges, 199 sont donc des archiereis ou archiereia. Les prêtres (hiereus/ hiereia), très minoritaires, sont chargés du culte de membres de la famille impériale dont le nom apparaît dans la titulature du sacerdoce, et non du culte impérial en général, à quelques rares exceptions près. ${ }^{6}$

Cette très forte homogénéité est le produit d'une histoire qui a simplifié et rendu l'objet du culte en quelque sorte abstrait: on honore les Augustes dans leur ensemble, et non plus des personnages précisément identifiés. Or, l’organe qui a initié ce mouvement de généralisation du culte est vraisemblablement l'assemblée provinciale, le koinon d'Asie. Certes, l'institution de la grande-prêtrise existe à l'époque hellénistique pour les cultes royaux puis pour certains cultes civiques, à Pergame et peut-être à Éphèse dans le deuxième tiers du I ${ }^{\text {er }}$ siècle a. C. ${ }^{7}$ Mais il est très probable que la spécialisation de ce titre dans le culte impérial soit une initiative des membres de l'assemblée d'Asie dès les années 20 a. C. L'évolution de la titulature de ce grand-prêtre provinciale est bien connue en particulier notamment grâce aux travaux de D. Campanile: à l'époque augustéenne, le grand-prêtre, élu annuellement par le koinon, porte le titre de „grand-prêtre de Rome et d'Auguste“, avec parfois l'ajout des titres du prince tels que père de la patrie. ${ }^{8}$ Dans les cités d'Asie, les grandes-prêtrises apparaissent très tôt et, souvent, elles sont occupées par des personnages de haut rang, dont beaucoup ont la citoyenneté romaine. À une date aussi haute, c'est le signe de leur appartenance à un cercle de notables de dimension provinciale. Ainsi, C. Iulius Épicratès de Milet a été archiereus du koinon d'Asie, de Milet et du koinon d'Ionie: même si rien n'indique qu'il ait été le fondateur de ces grandes-prêtrises à trois niveaux politiques différents, il est très probable qu'il fait partie d'un groupe de grands notables, héritiers des

5 Voir la prosopographie de ces prêtres sur www.pretres-civiques.org (ici abrégé $P C$ ) et l'étude que je leur ai consacrés (FRIJA 2012).

6 Prêtres de personnages honorés individuellement et non dans le cadre du culte collectif: voir par exemple $P C \mathrm{n}^{\circ} 53$ (Paros), 175 (Milet) et 76 (Ilion) pour Hadrien; 370-371 (Stratonicée de Carie) pour Agrippa; 101 (Pergame) pour Faustine. Prêtres des Augustes en général: $P C \mathrm{n}^{\circ} 109$ (Perperene), 197 (Attaleia), 102 (Pergame).

7 Sur l'assemblée provinciale à la fin de la République, voir CAmpanile 2007. Sur l'évolution de la grande-prêtrise à la fin de l'époque républicaine, FRIJA 2010; sur le cas d'Éphèse dans les années 30 a. C., KIRBIHLER 2012.

8 CAmpanile 1994. 
grands évergètes de la fin du $\mathrm{II}^{\mathrm{e}}$ siècle et de la première moitié du $\mathrm{I}^{\mathrm{er}}$ siècle $\mathrm{a}$. C, qui ont, dans le nouveau contexte politique créé par la victoire d'Octavien à Actium, mis en place et diffusé la grande-prêtrise du koinon vers les cités. Ils se sont ainsi ménagé un nouveau domaine d'action dans leur cité, tout en marquant leur loyauté personnelle et celle de leur communauté envers Auguste. ${ }^{9}$

Ce phénomène de diffusion d'une institution provinciale vers les cités se retrouve en deux autres occasions importantes en Asie. D'abord, c'est le koinon qui, le premier, adapte la titulature de son grand-prêtre à la situation nouvelle créée par la mort d'Auguste: le „grand-prêtre de Rome et d’Auguste“ devient „grand-prêtre“, sans autre précision. Il est probablement, dès ce moment-là, chargé du culte de Tibère et d'Auguste. Or, cette simplification apparaît aussi dans les cités sous le règne de Tibère et elle se diffuse progressivement, ce qui aboutit, dans le courant du I ${ }^{\mathrm{er}}$ siècle, à l'homogénéisation des titres présentées plus haut: le culte impérial civique est, partout, devenu un culte collectif des Augustes assuré par un grand-prêtre. ${ }^{10}$

La deuxième occasion importante dans laquelle le koinon apparaît comme le centre créateur d'une innovation diffusée ensuite dans les cités est la création de la grande-prêtrise féminine. Dans le koinon, l'apparition de l'archiereia suit la divinisation de Drusilla en 38 p. C., la première grande-prêtresse provinciale, Iulianè, épouse d'Alkiphron, étant explicitement mentionnée comme telle dans une inscription. ${ }^{11}$ Cette création est la réponse des Grecs d'Asie à un événement romain. Or, dans les cités, des femmes étaient impliquées dans le culte bien avant la divinisation de Drusilla: Auguste et Tibère ont eu des prêtresses dans certaines cités. ${ }^{12}$ Mais les premières grandes-prêtresses semblent postérieures à Caligula - quoi que ce point mérite de rester prudent, étant donné la fragilité des arguments $e$ silentio dans le domaine des études épigraphiques. ${ }^{13}$ Par la suite, l'archiereia féminine se diffuse largement dans

9 Sur les débuts du culte impérial dans les cités d'Asie, voir FrIJA 2012, 34-41.

10 Sur le rythme de ce phénomène, FrIJA 2012, 49-52.

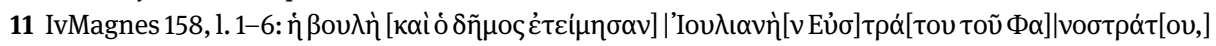

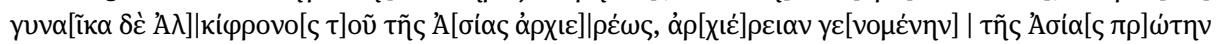

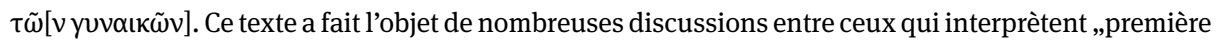
des femmes“ dans un sens honorifique et ceux qui le lisent comme une mention chronologique. Je me range ici à l'interprétation chronologique qui me paraît avoir été défendue de façon très convaincante par plusieurs savants (voire en particulier HERz 1992 et CAMPANILE 1994 n 8a). L'interprétation de la

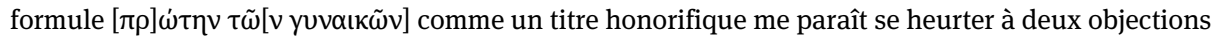
majeures: la date, très haute pour ce type d'usage, et l'organisation du texte, l'absence d'article devant

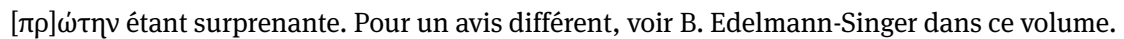

12 Voir par exemple $P C \mathrm{n}^{\circ} 5$ (Minnis, prêtresse de Tibère à Cos).

13 Pour des exemples, voir FRIJA 2012, 64-65 et 82-88. Un texte cependant pourrait remettre en cause la chronologie proposée ici: à Akmonia, Tatia fille de Menocritos, est grande-prêtresse dans une inscription datée par l'éditeur de 6/7 p. C. - le texte mentionne l'année 91 (THonEmANN 2010). Mais cette datation mérite un examen attentif que je ne peux mener ici. L’ère de Sylla, reprise par THONEMANN sans exprimer de doute, est proposée avec des points d'interrogation par LESCHHORN qui 
toute la province d'Asie. Il est donc probable que les dirigeants des cités ont créé une charge civique prestigieuse en imitation de celle que le koinon avait instaurée.

La diffusion de ces institutions du koinon vers les cités crée des cultes impériaux civiques très homogènes dans leurs structures. Mais le niveau social des grandsprêtres civiques et provinciaux reste très hétérogène, une fois passée la période de fondation: les grands-prêtres civiques sont essentiellement des notables de dimension locale. Cependant, leur comportement est calqué sur celui des grands notables de dimension provinciale. En compétition les uns avec les autres dans la cité, dans le contexte d'une compétition de plus grande ampleur entre cités, ils ont tendance à s’imiter les uns les autres et à produire de l'homogénéité.

Retrouve-t-on cette même homogénéité dans les autres manifestations du culte impérial et peut-on l'expliquer par les mêmes mécanismes sociaux et politiques? Il faudrait étudier cette vaste question dans chaque domaine de l'ensemble de rites que l'on regroupe sous le terme de „culte impérial“. Les résultats seraient peut-être différents dans le domaine des concours et des fêtes. Mais force est de constater que le koinon cherche à exercer une influence dans l'organisation des cultes civiques et que, loin de se contenter d'être le représentant des cités auprès de Rome, il se comporte, dans une certaine mesure, comme un dirigeant de la province. C'est notamment le cas lors de la réforme des calendriers civiques adoptée par le koinon en 9 a. C., suite à une proposition du proconsul Paulus Fabius Maximus: ${ }^{14}$ d'après ce texte célèbre, les cités décident dans le cadre du koinon d'adopter comme premier jour de l'année le jour anniversaire d'Auguste, le 23 septembre. En d'autres termes, le koinon adopte une mesure d'homogénéisation des calendriers civiques. De même, dans un document très mutilé connu notamment par une copie d'Halicarnasse, le koinon demande aux prêtres et grands-prêtres d'appliquer une décision commune qui a trait au culte impérial. L'état du texte ne permet malheureusement pas d'en savoir plus. ${ }^{15}$

Le koinon d’Asie a donc un poids, une influence sur les cités, parce que les cités sont en compétition, qu'elles s'observent et qu'elles s'imitent. C'est la compétition qui crée de l'imitation et, paradoxalement, de l'homogénéité.

évoque une autre possibilité: la cité aurait adopté l'ère de Pharsale, également attestée dans la région (LeschHorn 1993, 263-264). Dans ce cas, la prêtresse serait active dans les années 40. Cependant, l'inscription publiée par THonemann évoque un Cratès fils de Menocritos, peut-être le frère de Tatia, qui pourrait être le responsable d'un monnayage de la fin de l'époque augustéenne (RPC I 3168); mais on ne peut exclure qu'il s'agisse d'un ascendant, tant les noms sont fréquents. Il me semble que le dossier ne peut être refermé pour l'instant. Si la datation de THONEMANN est correcte, il faut alors inverser le raisonnement: la grande-prêtrise féminine serait une création civique adoptée par le koinon avant de se diffuser largement dans les autres cités.

14 IK 69, 14 (= OGIS 458); RDGE 65; LAFFI 1967.

15 GIBM 894. 


\section{Comparaison avec d'autres provinces grecques}

Ce fonctionnement très caractéristique de la grande province asiatique ne se retrouve pas de façon aussi marquée dans les autres provinces grecques.

\subsection{Bithynie-Pont}

En Bithynie, le culte de César, de Rome et d'Auguste a été créé en même temps que celui de l'Asie, comme l'indique le célèbre récit de Dion Cassius. Mais Dion Cassius n'évoque que les temples et non les prêtrises. La chronologie de fondation de la prêtrise provinciale de Bithynie nous échappe largement, car les sources d'époque augustéenne sont extrêmement lacunaires. Il est en tout cas douteux qu'un archiereus ait été instauré en Bithynie en même temps qu'en Asie car, dans la documentation plus tardive, le terme utilisé pour désigner le dirigeant annuel du koinon est bithyniarque, et non „archiereus de Bithynie“. Or, en Asie, la désignation comme asiarque, attestée à l'époque républicaine, ne redevient courante qu'à la fin du Ir siècle. En Bithynie, au contraire, c'est le terme bithyniarque qui s'impose. ${ }^{16}$ Dans le Pont, au contraire, bien que les sources soient peu nombreuses et tardives, la situation semble plus proche de celle de l’Asie: on trouve de façon à peu près égale „pontarque“ et „archiereus du Pont". 17

On ne peut donc pas parler, pour la province double de Bithynie-Pont, d'une situation identique à celle de l'Asie, pour deux raisons. D’abord, il s'agit bien d'une province double et la question de l'identité „bithynienne-pontique“ ne se pose visiblement pas. Ensuite, même en raisonnant à l'échelle de chacun des deux koina, le vocabulaire n'est pas homogène et n'est pas strictement identique à celui de l'Asie.

Qu'en est-il des cultes civiques dans cette province? La rareté des sources incite à la prudence, mais il semble que l'on retrouve la divergence entre les deux parties de la province observée au niveau des cultes provinciaux. En Bithynie, l'existence de la grande-prêtrise civique est incertaine et, même si elle a existé, elle est restée marginale: des prêtrises simples se maintiennent jusqu'à la fin du II ${ }^{\mathrm{e}}$ siècle. ${ }^{18} \mathrm{~S}$ 'il est

16 FERnOUX 2004, 343 et suiv. Voir par exemple IK 27, 47.

17 MAREK 2003.

18 Selon Fernoux 2004, la grande-prêtrise civique n'existe pas en Bithynie et tous les archiereis, même lorsqu'il n’y a pas de précision „de la Bithynie“, sont des prêtres provinciaux. La plus grande prudence est nécessaire, car les sources sont peu nombreuses: j’ai repéré moins de dix hiereis à Nicomédie (TAM IV 43), Nicée (IK 9, 63), Calchedon (IK 20, 19), Kios (IK 29, 13), Prusias (IK 39, 13 et 20). 
possible que des grands-prêtres civiques aient été instaurés à Prusias ${ }^{19}$ et à Nicée, ${ }^{20}$ on ne peut pas parler d'une diffusion massive de cette institution. Le terme archiereus reste très rare en Bithynie tant pour la prêtrise du koinon que dans les cultes civiques. Cela constitue une nette différence avec le caractère courant et banal du terme dans l'Asie voisine. De même, l'implication des femmes dans le culte impérial semble plus anecdotique. Aucune archiereia ni même hiereia civique n'est attestée, et une seule archiereia provinciale est connue. ${ }^{21}$

Dans le Pont, en revanche, la grande-prêtrise est bien connue dans plusieurs cités à Amastris ${ }^{22}$, mais aussi à Abonouteichos, Amisos, Pompeiopolis, Sebastopolis. ${ }^{23}$ Et surtout, il n'y a pas, à ma connaissance, de prêtrises simples des Augustes pris collectivement. La situation semble donc très proche de celle de l'Asie et la province double de Bithynie-Pont n'a pas d'unité du point de vue de l'organisation des cultes civiques: les prêtrises simples dominent en Bithynie alors que les grandes-prêtrises se sont imposées dans le Pont.

\subsection{Lycie-Pamphylie}

La situation est encore différente en Lycie, provincialisée plus tard: comme en Asie, les cultes impériaux sont très homogènes, mais en ce qu'ils séparent nettement la grande-prêtrise, réservée au koinon, et les prêtrises simples des cités. Comme en Bithynie-Pont, la province double de Lycie-Pamphylie n'a pas d'unité de ce point de vue: les structures des cultes civiques semblent au contraire nettement diverger entre les deux parties de la province.

L'instauration de la grande-prêtrise provinciale lycienne est une conséquence de la provincialisation de 43 p. C. Comme l'a montré ReItzenstein, il existait à l'époque séleucide une grande-prêtrise royale mais le culte de Rome, fondé dès le II ${ }^{\mathrm{e}}$ siècle $\mathrm{a}$. $\mathrm{C}$. par une ligue lycienne antérieure à la conquête romaine de l'Asie Mineure, était sous

19 L'organisation du culte impérial à Prusias n'est en effet pas très claire. Voir ainsi IK 39, 24: il pourrait s'agir d'une grande-prêtrise civique. Le personnage n'est pas classé parmi les prêtres provinciaux par MAREK 2003, 66.

20 Voir IK 9, 116 pour C. Cassius Chrestus, presbuteros (c'est-à-dire membre d'une association d'anciens, peut-être de la gérousia), archiereus et sébastophantès (chargé de montrer les images impériales lors des cérémonies): rien ici n'indique explicitement que le culte impérial soit celui du koinon. Pourrait-il s'agir d'un grand-prêtrise civique? Cela me paraît possible.

21 IK 27, 53 et Claudia Saturnina (MAREK 2003, 67).

22 MAReK 1993, 158-159 nº 3 Amastris, sous Néron: le peuple honore deux personnages, „grandsprêtres" sans autre précision, dont le père a lui-même été grand-prêtre trois fois de suite. En revanche, au début du II ${ }^{\text {e }}$ siècle, T. Iulius Aquila, honoré par les cités du Pont, est explicitement qualifié de „grand-prêtre du Pont“ (MAREK 1993, 160 nr. 7 Amastris)

23 Voir par exemple MAREK 1993, 156 nº 3 Abonouteichos; IGRRP III 134 (Pompeiopolis); SEG 41, 1112 (Sebastopolis). 
l'autorité d'un prêtre. Dans la période qui sépare le traité romano-lycien de 46 a. C. et la provincialisation, des cultes de Rome et des empereurs existent déjà, au niveau civique comme au niveau fédéral; on connaît notamment une prêtrise „de l'ethnos commun des Lyciens“ pour Tibère. ${ }^{24}$ Mais ce n'est qu'après 43 et l'inclusion du koinon lycien dans le système provincial romain qu'est introduite une grande-prêtrise du koinon pour le culte impérial. ${ }^{25}$ Il s'agit clairement d'une imitation du fonctionnement de l'Asie voisine, bien plus que de la reprise d'une grande-prêtrise hellénistique locale. $^{26}$

$\mathrm{Au}$ niveau des cités lyciennes, la situation n'est pas du tout la même qu'en Asie, puisque l'on continue à trouver très couramment, pendant tout le Haut Empire, des prêtrises simples. L'imitation de l'Asie ne va donc pas jusqu'à importer la grande-prêtrise au niveau civique. De très nombreuses cités ont au contraire conservé des prêtrises des empereurs. ${ }^{27}$ La règle, en Lycie, est de continuer à utiliser le titre de prêtre dans les cultes civiques et de réserver celui de grand-prêtre au koinon. ${ }^{28} \mathrm{~A}$ Xanthos, la mention d'une grande-prêtrise de la cité pourrait se référer à une grande-prêtrise fédérale exercé au Létôon, où se trouvait un ethnikon Kaisareion. ${ }^{29}$

24 ReITZENSTEIN 2011. Prêtre de l'ethnos: IGRRP III 474.

25 Sur les début du culte impérial en Lycie, ainsi que sur la spécificité du culte de Rome qui, en Lycie, est fondé vers 167 mais n'est jamais absorbé par le culte impérial et perdure pendant toute l'époque impériale, voir également CAMPANILE dans ce volume.

26 Voir la démonstration en ce sens de REITZENSTEIN 2011, 20-25. Le premier grand-prêtre de Lycie, Eirénaios de Xanthos, a vécu à l'époque claudienne: Reitzenstein 2011, 35 et $166 \mathrm{n}^{\circ} 1$. Seuls deux autres grands-prêtres lyciens peuvent être situés avant l'époque flavienne. Ead., 44: „Die Einführung der Archierosyne ist damit offenbar fest mit dem Konzept der römischen Provinzeinrichtung verbunden“. Je ne rentre pas dans le débat sur l'identité de l'archiereus et du lykiarque; comme en Asie, on trouve les deux termes, le terme lykiarque désignant probablement les archiereis en fonction, mais aussi ceux qui l'ont été dans le passé (Reitzenstein 2011, 53).

27 C'est le cas par exemple de Patara, Phaselis, Boubon, Oinoanda. Voir ainsi TAM II 189: le texte distingue clairement la grande-prêtrise fédérale et la prêtrise civique.

28 En ce sens, ReitZenstein 2011, 38. Il reste cependant quelques problèmes, par exemple dans TAM II 206 (Sidyma): une femme est honoree par le koinon et dite archiereia, sans précision du cadre dans lequel elle exerce sa grande-prêtrise. Il faut sans doute comprendre qu'elle est archiereia de Lycie, mais notons qu'en Asie, l'omission du nom de la province est extrêmement rare, même quand le contexte peut sembler clair. Un autre cas douteux dans TAM II 495. Avant la provincialisation, voir aussi le cas incertain de Polyperchon à Patara évoqué par ReITZEnstein 2011, 38 (TAM II 420): il est difficile de déterminer s'il s'agit d'un grand-prêtre civique ou fédéral.

29 BALLAND écrivait à ce sujet qu'une grande-prêtrise municipale était fort peu probable: „la documentation épigraphique, abondante sur ce point, paraît bien montrer qu'en Lycie les sacerdoces du culte impérial dans les cités sont occupés par des prêtres et prêtresses, non par des grands-prêtres et grandes-prêtresses“ (BALLAND 1981, 236) et, dans le même sens, Reitzenstein 2011 nº 105.1 Le cas est trop isolé pour en déduire l'existence d'une grande-prêtrise civique à Xanthos. En outre, on connaît un „prêtre des Augustes“ (TAM II 287, non daté; voir aussi FdXanthos VII 90), proche que ce qui existe dans le autres cités lyciennes. Le texte pourrait donc exprimer le fait que cette femme, une Xanthienne, a exercé le sacerdoce fédéral aussi dans la cité, puisqu'il y avait un sanctuaire lycien. 
En revanche, en Pamphylie, les grandes-prêtrises civiques sont tout à fait courantes et banales, comme en Asie: elles sont attestées à Pergé, Sidé, dans la petite cité de Kolybrassos, à Sillyon et Attaleia. ${ }^{30}$ Une archiereia apparaît dans les inscriptions de Sillyon. ${ }^{31}$ En revanche, aucun prêtre simple des Augustes n'est attesté: la situation est donc inverse de celle de la Lycie. Cela peut s'expliquer très simplement par le fait que la Pamphylie faisait partie de l'Asie à l'époque républicaine puis a été rattachée à la Galatie à l'époque augustéenne. Des grandes-prêtrises y ont été fondées en même temps que dans le reste de la province d'Asie, et n'ont pas été supprimées lors du rattachement à la Galatie ni lors de l'organisation de la double province - le koinon lycien n'ayant en outre autorité que sur les cités lyciennes.

Ailleurs en Anatolie, l'enquête sur l'organisation des cultes civiques reste encore largement à mener, mais le faible nombre d'inscriptions rend toute conclusion sur l'inexistence de telle ou telle institution très fragile.

\subsection{Achaïe}

En revanche, la comparaison avec l'Achaïe s'avère très intéressante sur plusieurs points. En premier lieu, les grandes-prêtrises civiques y apparaissent avant la fondation d'un culte fédéral. Ainsi, un archiereus est actif à Athènes sous Tibère, à un moment où les koina d'Achaïe n'assurent pas encore de culte impérial commun. ${ }^{32}$ Il est tentant d'en déduire que les cités de Grèce continentale ont emprunté cette structure de l'autre côté de la mer Égée, en Asie.

Un deuxième point intéressant est la disparition des prêtrises simples des Augustes dans les cités d'Achaïe au cours du Ir siècle. Au $\mathrm{II}^{\mathrm{e}}$ siècle, la grande-prêtrise civique semble avoir été adoptée partout: elle est attestée à Athènes, Sparte, Messène, Elis, Mégare, Thespies et Thèbes. ${ }^{33}$ En revanche, les grandes-prêtrises fédérales n'ont jamais eu le même poids qu'en Asie. Ce sont plutôt les cités qui sont créatrices d'institutions nouvelles. Il existe certes, à partir de Néron, des grandes-prêtrises fédé-

30 IK 54, 111 (Side Kitabeleri,); BEAN-MITTFORD 1962-63, 14; LANCKORONSKI 1890-1892, I 177 nº 60; IGRRP III 781 (Attaleia).

31 IGRRP III 802.

32 IG II 5034 et KANTIRÉA 2007, 61: l'inscription, gravée sur un siège du théâtre de Dionysos, a été corrigée de hiereôs Sebastou Kaisaros en hiereôs kai archiereôs Sebastou Kaisaros sous le règne de Tibère.

33 Voir les listes établies par CAMIA 2011: on connaît à Athènes au IIe siècle cinq grands-prêtres civiques et deux prêtres de Drusus. L'organisation est donc la même que dans les cités d'Asie où, à la grande-prêtrise générique, peuvent s'ajouter des prêtrises vouées à des personnages particulièrement honorés. 
rales et, au II ${ }^{\mathrm{e}}$ siècle, une helladarchie. ${ }^{34} \mathrm{Si}$ le résultat final, l'homogénéité des cultes civiques, est identique à la situation en Asie, ce résultat a été atteint par un processus inverse, dans lequel les cités sont à l'origine des innovations, alors que les koina ont peu de poids sur les cités. Le ressort fondamental, la concurrence entre cités, est cependant sans doute le même: si Sparte a une grande-prêtrise, les notables athéniens éprouvent certainement le besoin d'en créer une aussi. Mais la compétition se déroule directement entre cités, sans la médiation du koinon.

\subsection{Remarques sur la différence entre prêtrise et grande-prêtrise}

Avant de conclure, il convient de revenir sur les raisons qui conduisent à prêter une telle attention à la différence entre prêtrise et grande-prêtrise. Cette nuance institutionnelle a-t-elle une importance, dans la mesure où l'on constate une diffusion générale du culte impérial sur un mode commun à tout le monde grec, celui des cultes collectifs des Sebastoi? En effet, cette caractéristique constitue un élément d'unité et d'homogénéité incontestable. En Asie, elle trouve vraisemblablement son origine dans l'extension du culte à Tibère en 14 p. C.: en transformant le culte d'Auguste en culte des Augustes, les cités d’Asie pouvaient intégrer Tibère sans desservir Auguste. Peu à peu, la notion générique de Sebastoi permet d'honorer l'ensemble de la famille impériale, sans devoir en préciser la liste ni la limiter aux personnages reconnus comme divi par le Sénat. Or, il est bien connu que les Grecs n'ont pas traduit le terme divus, mais ont classé les empereurs parmi les Theoi. Le culte collectif permet de regrouper toutes ces entités divines dans un même ensemble. Il faut cependant préciser que le culte de la famille impériale - oikos, genos - n’a pas été adopté partout: il est quasiment inexistant en Asie, alors qu'il est bien attesté à Athènes et dans plusieurs autres cités d'Achaïe. Le culte des Sebastoi est à la fois plus vaste et moins précis que du genos ou de l'oikos des Augustes.

Pour en revenir à la différence entre prêtrise et grande-prêtrise, il ne me semble pas qu'elle doive être restreinte à une question de vocabulaire. En effet, la grande-prêtrise est un titre réservé au culte impérial. Elle est donc distincte des autres prêtrises de la cité, sans même qu'il soit nécessaire de préciser à qui elle est vouée. Ce simple fait place le culte impérial dans une position unique au sein des cultes civiques: le grand-prêtre est distingué des autres prêtres. En outre, grand-prêtre est un titre prestigieux: archiereus est le terme utilisé pour désigner le grand pontife romain, archiereus megistos, qui n'est autre que l'empereur lui-même. Il est issu d'institutions royales hellénistiques et c'est le titre que porte le dirigeant du koinon d'Asie. Archiereus a une connotation hiérarchique qui constitue une rupture par rapport à la tradition civique

34 Sur la nature de l'helladarchie, voir le compte-rendu de CAMIA sur l'ouvrage de LozANo 2010 (CAMia 2012). 
grecque dans laquelle les prêtres ne sont pas explicitement hiérarchisés. ${ }^{35}$ Pour toutes ces raisons, que la modeste cité d'Alioi en Phrygie ait un grand-prêtre et pas Tlos en Lycie ou Nicée en Bithynie ne me paraît pas un point de détail.

Enfin, l'étude du titre de grand-prêtre est un point d'entrée accessible pour se pencher sur la question de l'homogénéité des cultes civiques; et c'est d'ailleurs comme cela que je l'ai utilisé dans cette étude. Il serait nécessaire de mener l'enquête sur d'autres critères qui nécessiteraient des prosopographies par province, par exemple sur le caractère annuel ou viager de la prêtrise, son lien avec les agonothésies ou encore avec les magistratures éponymes des cités.

\section{Conclusion}

Peut-on proposer des pistes d'explication aux différences de pratiques entre provinces? L'histoire des différents koina semble avoir un poids important. Parmi eux, le koinon d'Asie a le plus fort poids politique, depuis l'époque républicaine: il a émergé pour la défense commune des cités. Mais la province d'Asie est aussi celle qui regroupe le plus grand nombre de cités puissantes, entre lesquelles il est difficile de trouver un équilibre: l'inflation des titres serait ainsi un résultat d'une compétition particulièrement forte. Inversement, le koinon lycien est peut-être davantage reconnu comme une instance supérieure aux cités; il serait plutôt question en Lycie d'une structure hiérarchique entre le koinon et la cité qui fait qu'on ne peut pas chercher à concurrencer le koinon, institution soudée et cohérente, outil de négociation commune davantage que lieu de confrontation et de concurrence entre les cités. Ces hypothèses restent à approfondir. Cette modeste étude de cas montre cependant que les rapports entre l'assemblée fédérale et les cités diffèrent profondément selon les provinces. L'autorité du koinon d'Asie peut paraître plus forte au premier abord, mais l'homogénéité de la province est surtout le produit d'une compétition encore plus forte: chacun doit avoir sa grande-prêtrise. En ce sens, on peut bien parler de spécificités provinciales, parce que le cadre romain a finalement eu un effet sur l'adoption ou le rejet de certaines institutions, sans pour autant que cela entraîne la formation d'identités provinciales revendiquées. ${ }^{36}$

Il faut enfin souligner, à un autre niveau, que ce sont plutôt les koina qui s'imitent les uns les autres que les cités. Les institutions ne circulent pas nécessairement à l'échelle de tout le „monde grec“. La notion de culte impérial „grec“ reste donc à affiner: les habitudes et spécificités régionales perdurent pendant tout le Haut Empire. Les structures complexes du culte impérial illustrent donc la complexité,

35 Sur les résonnances du terme, voir FRIJA 2010.

36 Ainsi, FERnoux 2013 conclut à l'absence d'identité provinciale proclamée. Mais cela n'empêche pas la formation de spécificités provinciales de fait, qui constituent une forme d'identité. 
mais aussi l'importance des phénonèmes „supra-civiques“ à l'époque impériale. Il ne suffit pas de raisonner à partir de deux éléments, les cités et Rome, ou „les Grecs“ et Rome: il faut également prendre en compte les subdivisions provinciales, les cadres administratifs romains. Cette démarche est indispensable pour affiner la caractérisation générique de „grec“, certes très utile et très juste à un certain niveau, mais trop englobante pour décrire la diversité de l'histoire de ces régions.

\section{Bibliographie}

BALLAND 1981 = A. BALLAND, Fouilles de Xanthos, Tome VII: Inscriptions d'époque impériale du Létôon, Paris 1981.

CAMIA 2011 = F. CAMIA, Theoi Sebastoi: il culto degli imperatori romani in Grecia, Provincia Achaia, nel secondo secolo D.C., Athènes/Paris, 2011.

CAMIA 2012 = F. CAMIA, Un culto imperiale 'provinciale' in Achaia? Riflessioni intorno a F. Lozano Gómez, Un dios entre los hombres. La adoración a los emperadores romanos en Grecia, Annuario della Scuola Archeologica Italiana di Atene e delle Missioni Italiane in Oriente 90, 2012, 351-358.

CAMPANILE 1994 = D. CAMPANILE, I sacerdoti del koinon d'Asia (I sec. a. C.-III sec. d. C.): contributo allo studio della romanizzazione delle élites provinciali nell'Oriente greco, Pisa 1994.

CAMPANILE 2007 = D. CAMPANILE, L'assemblea provinciale d'Asia in età repubblicana, in: G. URSo (ed.), Tra Oriente e Occidente: indigeni, Greci e Romani in Asia Minore. Atti del convegno internazionale, Cividale del Friuli, 28-30 settembre 2006, Pisa 2007, 129-140.

FERnoux 2004 = H.-L. Fernoux, Notables et élites des cités de Bithynie aux époques hellénistique et romaine (IIle siècle av. J.-C.-Ille siècle ap. J.-C.). Essai d'histoire sociale, Lyon 2004.

FERnOUX 2013 = H.-L. Fernoux, Bithyniens et Grecs d'Asie: à propos de la notion d'identité provinciale en Asie mineure sous le Haut-Empire, in: S. LefEBVRE (éd.), Identités et dynamiques provinciales du lle siècle avant notre ère à l'époque julio-claudienne, Dijon 2013, 61-86.

FRIJA $2010=$ G. FRIJA, Du prêtre du roi au prêtre de Rome et au grand-prêtre d'Auguste: mise en place des prêtrises de l'Empereur dans les cités d'Asie, in: I. Savalli-Lestrade/I. Cogitore (éd.), Des Rois au Prince. Pratiques du pouvoir monarchique dans l'Orient hellénistique et romain, Actes de la table ronde organisée à l'INHA (Paris) les 23 et 24 mai 2008, Grenoble 2010, 291-308.

FRIJA $2012=$ G. FRIJA, Les prêtres des empereurs: le culte impérial civique dans la province romaine d'Asie, Rennes 2012.

FuJII $2013=$ T. FuJII, Imperial Cult and Imperial Representation in Roman Cyprus, Stuttgart 2013.

GIBM = C.T. Newton/G. Hirschfeld/ F.H. Marshall (ed.), The collection of Ancient Greek Inscriptions in the British Museum, Oxford, 1874-1916.

GRADEL $2002=$ I. GRADEL, Emperor Worship and Roman Religion, Oxford 2002.

HARLAND $2003=$ P. HARLAND, Associations, Synagogues, and Congregations: Claiming a Place in Ancient Mediterranean Society, Minneapolis 2003.

HERz 1992 = P. Herz, Asiarchen und Archiereiai: Zum Provinzialkult der Provinz Asia, Tyche 7, 1992, 93-115.

KANTIRÉA $2007=$ M. KANTIRÉA, Les dieux et les dieux augustes: le culte impérial en Grèce sous les Julio-claudiens et les Flaviens, Athènes 2007.

KIRBIHLER 2012 = F. KIRBIHLER, César, Auguste et l'Asie: continuités et évolutions de deux politiques, in: O. Devillers/K. Sion-Jenkis (éd.), César sous Auguste, Bordeaux 2012, 125-144.

LAFFI $1967=$ U. LAFFI, Le iscrizioni relative all'introduzione nel 9 a. C. del nuovo calendario della Provincia d'Asia, Studi classici e orientali XVI, 1967, 5-98. 
LANCKoronski 1890-1892 = K. LANCKoronski/G. Niemann/E. Petersen, Städte Pamphyliens und Pisidiens, Prague/Vienne/Leipzig, 1890-1892.

LePeLLey 1998 = C. LePelley (éd.), Rome et l'intégration de l'Empire, 2. Approches régionales du Haut-Empire romain, Paris 1998.

LESCHHORN 1993 = W. LeSCHhoRn, Antike Ären: Zeitrechnung, Politik und Geschichte im Schwarzmeerraum und in Kleinasien nördlich des Tauros, Stuttgart 1993.

LOZANO $2010=$ F. LOZANO, Un dios entre los hombres: la adoración a los emperadores romanos en Grecia, Barcelona 2010.

MAREK 1993 = C. MARek, Stadt, Ära und Territorium in Pontus-Bithynia und Nord-Galatia, Tübingen 1993.

MAReK 2003 = C. MAReK, Pontus et Bithynia: Die römischen Provinzen im Norden Kleinasiens, Mainz 2003.

MAREK $2010=$ C. MAREK, Geschichte Kleinasiens in der Antike, München 2010.

PRICE 1984 = S. PRICE, Rituals and Power: The Roman Imperial Cult in Asia Minor, Cambridge 1984. REITZENSTEIN 2011 = D. REITZENSTEIN, Die lykischen Bundespriester: Repräsentation der kaiserzeitlichen Elite Lykiens, Berlin 2011.

SARTRE 1997 = M. SARTRE, Les provinces de Méditerranée orientale d'Auguste aux Sévères, 31 av. J.-C. -235 apr. J.-C., Paris 1997.

SARTRE $1998=$ M. SARTRE, L'Orient sémitique, in: LePELLEY 1998, 333-339.

Thonemann $2010=$ P. Thonemann, The Women of Akmoneia, JRS 100, 2010, 163-178. 